\title{
Labyrinthe
}

41 | 2014-2015

Ici la Dédalie

\section{Fictions et visions littéraires d'un bateau négrier. Penser en ethnographe le conte Benito Cereno d'Herman Melville}

\section{Gaetano Ciarcia}

\section{OpenEdition}

Journals

Édition électronique

URL : http://journals.openedition.org/labyrinthe/4365

DOI : $10.4000 /$ labyrinthe.4365

ISSN : 1950-6031

Éditeur

Hermann

\section{Édition imprimée}

Date de publication : 1 avril 2015

Pagination : 91-105

ISBN : 9782705690717

\section{Référence électronique}

Gaetano Ciarcia, «Fictions et visions littéraires d'un bateau négrier. Penser en ethnographe le conte Benito Cereno d'Herman Melville », Labyrinthe [En ligne], 41 | 2014-2015, mis en ligne le 03 juillet 2015, consulté le 10 décembre 2020. URL : http://journals.openedition.org/labyrinthe/4365; DOI : https:// doi.org/10.4000/labyrinthe.4365 


\title{
Fictions et visions littéraires d'un bateau négrier Penser en ethnographe le conte Benito Cereno d'Herman Melville
}

\author{
Gaetano Ciarcia \\ ciarcia.gaetano@wanadoo.fr
}

$\mathrm{Si}$ «presque toutes les œuvres de fiction marquantes transmettent un "message" ou des "messages" qui sont transmis par le texte mais ne sont pas dans le texte ${ }^{1} »$, comment les écritures romanesques génèrentelles des significations et des jugements sur la réalité ? Sans prétendre donner une réponse péremptoire à cette question, nous pouvons émettre l'hypothèse que lorsque nous imaginons des transferts sémantiques dont les figures d'un récit littéraire peuvent être les vecteurs, nous sommes confrontés à des suggestions susceptibles de se «prolonger ${ }^{2} »$ dans une connaissance anthropologique des faits sociaux et historiques. Ces fictions nous permettent parfois de penser autrement des relations, qui, tout en étant l'effet d'une altération narrative de la réalité, ne sont pas moins des miroirs (soient-ils déformants) ou des tableaux (soient-ils cryptés) d'un contexte, d'une époque, d'un milieu. À partir de ce point de vue, les objets et les personnages de la nouvelle Benito Cereno de Herman Melville ont inspiré ma réflexion sur des formes de confrontation dramatiquement théâtrale à l'altérité et sur la question de la présence à soi de leur observateur-acteur ${ }^{3}$.

1. John R. Searle, Sens et expression : études de théorie des actes de langage (trad. de l'anglais par J. Proust), Paris, Éditions de Minuit, 1982 [1979], p. 118.

2. Pierre Bayard, Peut-on appliquer la littérature à la psychanalyse?, Paris, Éditions de Minuit, 2004 : 152. Selon Bayard, prolonger un texte consisterait à « relever un certain nombre des éléments de pensée qui y figurent directement ou à l'état de virtualités, de les réunir et d'organiser avec eux une réflexion théorique ». En suivant cette piste, il vise le renversement de la relation entre littérature et psychanalyse, à travers une attention pour l'œuvre beaucoup moins comme un contexte à interpréter que comme un monde fictif questionnant tacitement les catégories d'une discours institué.

3. Herman Melville, Benito Cereno (trad. de l'anglais par J.-P. Naugrette), Paris, Flammarion, 1991 [1855]. Pour une présentation détaillée de la trame de ce conte qu'ici ne sera qu'évoquée, je renvoie le 
C'est l'histoire, imaginée par Herman Melville avoir eu lieu en 1799, près de l'îlot désertique de Santa-Maria au large du Chili, du capitaine Amasa Delano qui ne s'aperçoit pas que sur le San Dominick - un bateau négrier espagnol en détresse à qui il porte secours - les Blancs sont devenus les otages des Noirs. Il se rend bien compte que règne sur le navire une inquiétante étrangeté, mais le code pour l'interpréter lui manque. L'intrus, se voulant être le sauveur, est en réalité la dupe que Benito Cereno, le capitaine du San Dominick, lui-même tenu en otage, tente désespérément de protéger. Delano essaie pourtant de maîtriser les signes à la fois familiers et incongrus à travers des hypothèses diverses par moments il imagine Benito Cereno en ennemi, chef d'un équipage de pirates - qui se révèlent être autant des illusions nécessaires à sa survie que des convictions funestes.

L'intrigue melvillienne explore les objets concrets de l'observation en les transformant en images iconiques, comme si elles étaient un aperçu de ce qui dans toute enquête, en tant que tentative d'objectivation d'une vérité, reste toujours encore à penser. De ce fait, le «sens du réel» dont l'écrivain fait preuve s'exprime aussi à travers les imaginaires historique et géographique que ces objets (dotés d'une efficacité à la fois représentative de mondes sociaux et prédictive de la trame du récit) éveillent chez le lecteur. Cette qualité est présente dans toute l'œuvre de Melville; par exemple, dans Moby Dick, lorsque Ishmael raconte son passage au Spouter-Inn à New Bedford où il attend le départ du bateau pour Nantucket, à l'entrée de l'auberge, parmi les vestiges des épopées marines vécues par ses hôtes, il observe une panoplie d'armes «païennes»; mais, c'est surtout une peinture crasseuse - un sombre présage de l'histoire à venir, voire «un commentaire, un résumé de Moby Dick même ${ }^{4} »-$ qui capte son attention : on y aperçoit les images d'un navire en proie aux vagues du Cap Horn et d'une baleine empalée sur ses trois mâts. D'après Howard P. Vincent, «Il n'y a pas jusqu'aux dessins grossiers gravés sur le doublon (chapitre 99) [de Moby Dick] qui ne remplissent pas une fonction extra-narrative. Il en va de même de l'idole païenne (chapitre 24) de Tä̈pi, du navire en verre dans Redburn et du portrait [du père] dans Pierre $^{5} »$. Cette remarque peut

lecteur à mon texte « L'objet invisible, ou le gambit du Capitaine », L'Homme, 170 (2004), p. 181-190, d'où je reprends le paragraphe qui suit.

4. Howard P. Vincent, «Ishmael, écrivain et critique d'art », L'Arc, 41 (1970), p. 48.

5. Ibid., p. 49. 


\section{Fictions et visions littéraires d'un bateau négrier}

s'appliquer à Benito Cereno où des objets du récit - dont la signification échappe ou se cache à l'observateur protagoniste de l'histoire, le capitaine Amasa Delano du navire Bachelor's Delight, - semblent exposer, tout en les voilant, l'évolution du scénario et la vérité à atteindre. Ces objets agissent comme des miroirs réverbérant un univers affecté par la traite négrière. Un monde est aperçu, mais aussi dissimulé, grâce aux choses qui le rappellent et qui le rendent anthropologiquement possible ${ }^{6}$.

Inspirée d'un fait qui s'est véritablement produit à l'époque de la traite négrière, l'enquête romanesque du protagoniste de Benito Cereno est ponctuée par des jeux de miroir, des mises en abîme, d'images aux signifiés flottants. Voici quelques exemples : la figure de proue du San Dominick, représentant un satyre noir masqué dont le pied est «posé sur le cou prostré d'une figure contorsionnée, également masquée ${ }^{7}$ » ; les faucilles que Delano remarque dans les mains libres de ceux qui devraient être les esclaves; le nœud qu'un des matelots demande de dénouer au capitaine américain qui s'est porté au secours du bâtiment espagnol; la clé (qui n'ouvre plus rien) attachée par une cordelette de soie au cou de Benito Cereno ; le collier de fer d'où pend une chaîne censée immobiliser le colosse Atufal (qui, en réalité, étant le geôlier discret de Cereno, est libre de ses mouvements); le fourreau de l'épée du même capitaine castillan qui a été raidi mais qui est vide en réalité ; le commentaire aux mots de craie : Suivez votre chef indiquant la carcasse de l'armateur et négrier Aranda exhibée - avant l'arrivée du Bachelor's Delight - par les esclaves révoltés afin d'effrayer l'équipage du San Dominick, puis cachée - durant la présence de Delano à bord -, enveloppée dans un linceul de toile; le drapeau royal espagnol utilisé par le serviteur noir Babo comme tablier pour raser son maître déchu Benito Cereno, en présence de Delano étonné.

6. À propos de l'opposition entre le « sens du réel » des écrivains théorisé par Émile Zola dans $L e$ Roman expérimental (1880), pour définir une qualité de l'écrivain doté d'une connaissance de la «nature » de la vie sociale, Jacques Bouveresse cite le texte de Cora Diamond (L'Esprit réaliste. Wittgenstein, la philosophie et l'esprit, trad. de l'anglais par Jean-Yves Mondon, Paris, PUF, 2004 [1991]) : «Les possibilités ne sont pas étalées à la surface des choses. Voir les possibilités des choses est une chose qui dépend d'une espèce de transformation de la perception que nous avons d'elles. Les possibilités ne se donnent que, pour ainsi dire, sous pression ». Bouveresse nous dit alors que « si on considère les choses de cette façon, on comprend sans doute un peu mieux [...] l'importance que Musil, dans L'Homme sans qualités (1930), accorde à ce qu'il appelle le "sens du possible" par opposition au "sens du réel" [...] » : Jacques Bouveresse, La Connaissance de l'écrivain. Sur la littérature, la vérité et la vie, Marseille, Agone, 2008, p. 144.

7. Herman Melville, op. cit., p. 65. 
De telles occurrences narratives sont les supports d'une invention littéraire qui «fonctionne» sémiotiquement comme cadre réaliste et allégorique à la fois d'une époque et de ses drames ${ }^{8}$. Dans le «nouveau monde» américain aux identités changeantes et échangeables que Melville explore dans son œuvre, ces apparitions expriment également l'invisibilité, voire l'impossibilité, d'un rapport stable et définitif entre les diverses figures du récit. Reprenant une formule proposée par William T. Lhamon Jr., nous pouvons concevoir ces figures comme des «talismans culturels ${ }^{9}$ », c'est-à-dire comme des entités dotées par l'écrivain d'une double efficacité initiatique : elles introduisent le lecteur dans l'histoire et en même temps elles préservent le sens ultime (le mystère) sous-jacent à leur nécessité narrative, voire la possibilité, toujours latente dans l'intrigue, de leur renversement sémantique : «Les histoires symboliques, comme les stéréotypes, sont des exemples de surdétermination compacte : leur énergie et leur assurance vont au-delà de leurs ressources apparentes. Elles véhiculent des réserves cachées ${ }^{10}{ }$.

Melville montre les signes d'un ordre violé. Leur opaque singularité s'oppose à la quête d'une inclinaison collective à rendre transparente, comme elle est pratiquée par le capitaine américain en mission de sauvetage. La «vérité» de la vicissitude, la révolte des esclaves, est invisible parce qu'elle est maquillée, d'une manière temporellement et spatialement originale, comme les objets sur lesquels la narration insiste. Détournées de toute mythologie domestique ou idéologie bourgeoise (celle dont le capitaine Delano est imprégné), au lieu d'être figées - comme si elles étaient «muséalisées » dans une vitrine ou sur un piédestal - les pièces

\footnotetext{
8. William T. Lhamon Jr. a fait remarquer que d'entrée de jeu le nom San Domininick lui-même est déjà métaphore de la révolution des esclaves dans l'île de Saint-Domingue (devenue plus tard la république noire d'Haïti) et l'année 1799 où l'action se situe est celle du premier traité signé par Toussaint Louverture avec la Grande-Bretagne et les États-Unis. Voir William T. Lhamon Jr., Raising Cain. Représentations du blackface de Jim Crow à Michael Jackson, trad. de l'anglais par Sophie Renaut, Paris, Kargo \& L'Éclat, 2004 [1998], p. 108. De son côté, Pierre Leyris avait émis une toute autre hypothèse : «Le navire tout entier est sous le signe d'un redoutable memento mori. Et que penser de son nom ? San Dominick répond évidemment à "frères noirs", appellation usuelle des dominicains. Mais il y a pour le moins une coïncidence singulière dans le rapport de ce nom à celui du capitaine castillan. On sait que l'on nommait san benito la casaque dont on revêtait ceux que l'Inquisition envoyait au bûcher. Si l'on songe au supplice que souffre Don Benito, à ses tortionnaires, et d'autre part au rôle que le dominicains jouèrent dans l'Inquisition, on à peine à croire que le rapprochement soit fortuit ». Voir Pierre Leyris, « Naissance des contes », L'Arc, 41 (1970), p. 62.

9. William T. Lhamon Jr., op. cit., p. 100.

10. Ibid., p. 101.
} 
du récit, évoluant devant l'observateur, ne sont pas seulement un produit de/pour son regard, mais, tout en étant presque impensables, avec la complicité de ses dénégations, s'imposent également à lui, en l'aveuglant.

\section{Les personnages-récit}

L'origine du conte est le recueil du capitaine Amasa Delano, navigateur et marchand américain (1763-1823), auteur du récit autobiographique Narrative of Voyages and Travels in the Northern and Southern Hemispheres (1817) relatant aussi de la révolte des esclaves qui avait eu lieu en 1805 à bord du navire espagnol Tryal (le San Dominick de la nouvelle, alors que le nom «véridique» du Bachelor's Delight était Perseverance). Pour ce qui concerne la réalité historique de la figure du vrai capitaine Benito Cereno, dans le document signé par Delano, qui complète son témoignage avec les actes juridiques du procès (repris et transformés à son tour par Melville lui-même) clôturant les événements, il apparaît sous le nom de Bonito Sereno ou de Benito Cereno. La modification romanesque de ce personnage et la chute des événements ont été presque complètement inventées par l'écrivain. Par exemple, dans la version originale établie par le vrai Delano, Cereno est peint comme un être haineux et ingrat ayant tenté de poignarder un prisonnier noir et n'ayant jamais voulu, par la suite, payer à Delano et à ses hommes la récompense promise avant la «reconquête» du Tryal. Il est intéressant de souligner aussi que, d'après l'instruction du procès, Cereno lui-même, qui était déjà leur otage, avait proposé aux mutins, pour les tranquilliser, de jouer le stratagème devant Delano.

Dans sa relation avec le témoignage du vrai Delano, la fiction interroge, en la transformant, la source documentaire plus que cette dernière éclaire l'histoire racontée par Melville. À travers la modification des événements et des caractères moraux des personnages, le texte illustre l'écroulement (Cereno) et l'édification (Delano) de deux systèmes légaux de croyances. Ainsi, l'altération de la source remplace la description du dispositif ordinaire relatif au maintien de la hiérarchie en vigueur. En ce sens, ce n'est pas l'instance finale du droit (le verdict du tribunal) que la confrontation aussi «raciale» de l'histoire élucide, mais plutôt la composition du conte qui semble expliquer la dialectique inhérente à la vérité issue du déroulement du procès. Comme l'a fait remarquer 
Susan Weiner, «in a text with a multitude of highly developed symbols, the ultimate symbole is law ${ }^{11} \gg$. En questionnant la liaison que la fiction entretient avec la loi comme forme de production de la croyance, Melville esquisse, à travers une ethnographie faussement naïve d'un microcosme spécifique (un bateau négrier à la dérive), une anthropologie imaginaire des effets de représentation au sein d'une société esclavagiste et coloniale.

Dans ce conte, tout comme les objets, les caractères humains apparaissent affectés par « une lente, lourde et louche sorcellerie ${ }^{12} »$. Par exemple, le rôle du maître subit une sorte d'envoûtement dont le personnage tragique de Benito Cereno - possédé, en quelque sorte, par celui qui fût son esclave et qui l'enchaîne à son ancienne condition de seigneur - en est la victime. De son côté, s'obstinant, à travers ses dénégations, à ne pas voir la révolte en acte malgré les comportements inexplicables vus à bord du San Dominick, le capitaine Amasa Delano semble obliger sa pensée à égarer quelques-uns de ses repères cognitifs immédiats pour sauvegarder des certitudes en sursis (en réalité, il ne s'agit désormais que d'illusions).

En écrivant une fiction sur les fictions du réel, «la plus grande ruse de Melville aura été sans doute d'inoculer chez ses lecteurs les germes de leurs propres préjugés ${ }^{13}{ }^{»}$. L'auteur de Benito Cereno porte son regard sur l'incapacité du capitaine Amasa Delano, mauvais lecteur de l'histoire mise en scène par les esclaves, à décrypter l'inversion des identités en cours dont les objets et les gestes qu'il observe sont les témoins muets. Delano, in vivo, tente de (a intérêt à) traiter la situation dans sa dimension supposée solidaire, alors que dans la réalité imaginée par Melville, elle est littéralement hors-la-loi, traversée et divisée par les conflits séparant les Blancs des Noirs, le seigneur déchu Benito Cereno du valet Babo qui apparemment le sert mais qui en réalité le domine. Ici les «lumières» de la croyance n'arrivent pas à éclairer les ténèbres de la réalité esclavagiste dans lequel tous les personnages du récit cherchent désespérément leur salut. Et pourtant, le seul à le trouver sera le crédule, celui qui de ces ténèbres n'a aperçu que l'ombre menaçante, sans rien y voir, sinon les oscillations de son optimisme conquérant. En déniant les manifestations

11. Susan Weiner, «"Benito Cereno" and the Failure of Law », Arizona Quarterly, 47 (1991), p. 25. 12. Colette Audry, «Compte rendu » de Benito Cereno et autres contes de la Veranda, Les Temps Modernes, 71 (1951), p. 573.

13. William T. Lhamon Jr., op. cit., p. 104. 


\section{Fictions et visions littéraires d'un bateau négrier}

visibles du danger, Delano prétend participer, innocemment et légalement, à la situation que Babo, la moitié «maudite» et cachée possédant Cereno (l'interlocuteur légitime, l'homologue en grade et en «race» de Delano), a désormais dé-domestiqué. D’ailleurs, la dissociation entre la présence ambigument candide du capitaine américain et l'ancien régime espagnol mourant, incarné par le souffrant Benito Cereno, est présente implicitement comme la différence fondatrice d'un nouvel ordre colonial.

\section{Une fable pour anthropologues?}

L'inaptitude à percevoir le drame dont il est l'enjeu et le spectateur ainsi que sa contemporanéité, dans le lieu et à travers les objets de sa manifestation immédiate, dénote le gouffre entre le regard fermé de Delano et les vécus diachroniques, désormais communs quoique férocement divisés, de Cereno et de Babo. Melville est l'ethnographe de leur intimité brutale et son conte est « une histoire d'œil qui montre, en même temps, le sujet et l'objet d'un acte de perception ${ }^{14} »$.

La rencontre vécue par Delano à bord du San Dominick illustre comment la fiction littéraire peut agir telle une «"variation imaginaire" des rapports sociaux ${ }^{15}$ » ayant pour objet moins l'étude d'un cas qui validerait une théorie qu'un lieu d'où l'invention romanesque interroge la fiction des réalités qu'elle fabrique en les observant. Melville fait subir à une des figures incarnant la position du regard et du commandement, le renversement de l'idée d'autorité et de l'étendue de l'oppression, mais aussi d'une hégémonie compréhensive : si «Delano n'a pas voyagé assez loin sous le rasoir de Babo, ni assez longtemps dans l'espace social extrêmement angoissant du cuddy du San Dominick, pour concevoir ce que peuvent ressentir les Espagnols. Cereno peut se faire une meilleure idée de Babo en raison de sa "complicité" dans la "farce" du rasage ${ }^{16} »$. À travers sa participation tragique, le capitaine espagnol a connu et occupé la place de l'Autre dans le théâtre de la domination; sa «complicité» dans la mascarade des esclaves a transmué radicalement et à jamais

14. Philippe Jaworski, « Notes de "Benito Cereno" », dans Herman Melville, Euvres IV : Bartleby le scribe, Billy Budd, marin et autres romans, Paris, NRF Gallimard, 2010, p. 1087.

15. Jean Jamin, « Fictions haut régime. Du théâtre vécu au mythe romanesque », L'Homme, 175-176 (2005), p. 196.

16. William T. Lhamon Jr., op. cit., p. 109. 
les repères qui lui étaient familiers et nécessaires à la pratique et aux représentations de son pouvoir.

Dans la scène du rasage, la machination procédant du contexte «exotique » investit de plein fouet la personne de l'observateur étranger qui tente vainement de conquérir son insertion intelligente parmi ses «hôtes ». Delano surprend Babo en train de raser Benito Cereno à l'aide d'une bien étrange serviette :

Le château et le lion! s'écria le capitaine Delano - mais Don Benito, c'est le pavillon espagnol dont vous vous servez là! il vaut mieux que ce soit moi seulement, et non le roi, qui soit témoin de cela, ajouta-til avec un sourire, mais - et il se tourna vers le Noir - c'est tout un, j'imagine, pourvu que les couleurs soient gaies; remarque plaisante qui ne manqua point de titiller le nègre ${ }^{17}$.

La présence du miroir donne à voir la comédie en cours où le secret se niche dans le rasoir manié du «barbier» Babo, dans le mutisme angoissé de son «client» Cereno et dans la mauvaise foi de Delano. Cette scène, absurde et révélatrice en même temps, a comme accoutrement le pavillon royal bafoué, utilisé par le «noir» farceur, amant des couleurs gaies bien sûr, comme tablier pour draper son «maître». Sur ce terrain dangereux, pour Delano, il s'agit de ne pas succomber à la singularité de la situation et de ses objets, là où à être invisible est moins la personne de l'intrus que l'identité antagoniste des esclaves, pourtant si concrète, observant et menaçant à son insu (?) l'observateur-protagoniste du récit. Confronté pendant toute la durée de son séjour, très court mais intense (de l'aube au crépuscule), à l'évidence de la révolte, Delano ne la voit qu'une première fois, au moment du dénouement final des événements, alors que Cereno, qui échappe à Babo se lançant dans le canot venu chercher le capitaine américain, est désormais pris par le monde d'ombres où l'atroce proximité de l'Autre l'a exilé à jamais :

- Vous êtes sauvé, s'écria le capitaine Delano, de plus en plus surpris et peiné : vous êtes sauvé; qu'est-ce qui a jeté une telle ombre sur vous?

- Le nègre.

17. Herman Melville, Benito Cereno, op. cit., p. 121. 


\section{Fictions et visions littéraires d'un bateau négrier}

La retraite spirituelle du capitaine espagnol dans un monastère, après la fin du procès à Lima, ne fait qu'entériner une mort sociale déjà advenue à bord du navire négrier et qu'aura anticipé de quelques mois sa disparition physique. Cereno va désormais «suivre son $\operatorname{chef}^{18}$ », non pas l'armateur Aranda tué durant la mutinerie, mais le nègre Babo dont la mort met fin aussi à l'histoire réécrite par Melville, comme s'il en avait été le protagoniste :

Quelques mois plus tard, traîné vers le gibet à la queue d'une mule, le Noir connut sa fin silencieuse. Le corps fut réduit en cendres; mais pendant longtemps, la tête, cette ruche de subtilité, fixée sur une perche dans la Plaza, soutint sans fléchir les regards des Blancs tandis que les yeux contemplaient par-delà la Plaza l'église Saint-Bartholomé, dans les caveaux de laquelle reposaient alors, comme à présent, les os recouvrés d'Aranda; et par-delà le pont Rimac, le monastère du mont Agonia, dans lequel, trois mois après avoir été renvoyé par la Cour, Benito Cereno porté en terre, ne manqua point, cette fois, de suivre son chef $^{19}$.

\section{De l'observation aveugle au théâtre des observés}

D'après Andrew Abbot, «La description [...] est toujours locale. Elle s'occupe d'un lieu géographique ou social, d'un ici, et aussi un lieu temporel, d'une époque actuelle, d'un maintenant ${ }^{20} \gg$. Toujours selon Abbot, «L'apparence d'immobilité relève de l'ignorance de la part de l'observateur des nombreux processus dont il ne voit que les extérieurs; les scandales, les maladies, les brouilles sont en cours, mais les renseignements manquent qui permettraient de les "voir" $[\ldots]^{21} »$. Nous pourrions en conclure qu'on ne voit que ce qu'on croit devoir comprendre; on ne

18. Ibid., p. 168. Avant l'arrivée de Delano, pour terroriser les matelots espagnols du San Dominick, les restes de l'armateur Aranda avaient été exposés durant la mutinerie (après que des esclaves ashanti en avaient dépecé le corps, lui rendant, sous forme de squelette, une blancheur effrayante) avec par commentaire de mots en craie « Suivez votre chef » en contrebas.

19. Ibid.

20. Andrew Abbott, « La description face à la temporalité », dans Giorgio Blundo et Jean-Pierre Olivier de Sardan (dir.), Pratiques de la description, Paris, Éditions de l'EHEss, 2003, p. 43.

21. Ibid., p. 45. 
voit que ce qu'on croit devoir interroger; on n'interroge pas ce qu'on ne veut/peut pas comprendre ou voir. Mais, Abbot poursuit : «[...] cette invisibilité momentanée des processus en cours est le parallèle exact de l'invisibilité des grandes structures sociales dans le lieu immédiat de l'ethnographe, pour qui elles sont présentes mais impossibles à distinguer des choses purement locales ${ }^{22} »$, donc, pour le sociologue américain, «ce qu'il faut expliquer, ce n'est pas le changement. [...] C'est la stabilité23». Cette stabilité rappelle le «calme plat» que Melville évoque entre autres dans son roman Mardi - en effet, il est question de «la terreur du calme plat»- mais également celui terrestre, auquel est confronté Tommo durant son séjour, à la fois doré et angoissé, chez les Taïpi, prétendus anthropophages de l'île de Nuku-Heva, dont il est l'hôte choyé mais aussi le prisonnier ${ }^{24}$ ou celui que le capitaine Delano scrute à bord et autour du San Dominick. Entre l'Eden cannibale de Taïpi et l'Enfer à bord du San Dominick, sur le thème de l'incapacité de penser l'Autre, épistémiquement absent, dans sa contemporanéité ${ }^{25}$, Melville agence des combinaisons variables de significations métaphoriques. À travers une «rhetoric of abuse ${ }^{26} »$, c'est une mise en crise de l'idéal de la simultanéité (et de la réciprocité) de la rencontre avec l'Autre que Melville pratique, faisant preuve, à travers son art, d'une réflexivité anthropologique avant la lettre.

Selon Freud, «un contenu refoulé de représentation ou de pensée peut percer jusqu'à la conscience, à condition qu'il se laisse dénier. La dénégation est une façon de prendre conscience du refoulé, elle est à proprement parler, déjà une levée du refoulement, mais, certes, pas une acceptation du refoulé ${ }^{27} \gg$. Cette acceptation étant un acte fondateur d'une croyance, l'objet refoulé peut (doit) être invoqué sous forme de fétiche, pour conjurer le danger qui serait constitué par la reconnaissance de la fiction. Si on reste au plus près du principe freudien, le fétiche nie, accepte et conserve une disparition; objet ethnographique et/ou métaphore catégorielle, le

22. Ibid., p. 46.

23. Ibid., p. 53.

24. Herman Melville, Taïpi (trad. de l'anglais par T. Varlet et F. Ledoux), Paris, Gallimard, 1952 [1846].

25. Cf. Johannes Fabian, Le Temps des autres. Comment l'anthropologue construit son objet, trad. de l'anglais par Estelle Henry-Bossoney et Bernard Müller, Toulouse, Anacharsis, 2006 [1983] ; Michel Foucault, L'Archéologie du savoir, Paris, NRF Gallimard, 1969.

26. Tony McGowan, « Suffering from simultaneity in "Benito Cereno" », $Q / W / E / R / T / Y, 11$ (2001), p. 109. 27. Sigmund Freud, La Dénégation (trad. de l'allemand par P. Thèves et B. This), Paris, Le Coq-Héron, 1982, p. 13. 


\section{Fictions et visions littéraires d'un bateau négrier}

fétiche est la dénégation, parce qu'il est précisément voué à préserver une croyance illusoire de sa désagrégation ${ }^{28}$. Dans Benito Cereno, les pièces «impossibles » du récit aperçues par l'observateur semblent esquisser, à travers leur ambivalence de signes, une cosmogonie paradoxale où les objets sont des choses imagées, des «fétiches », c'est-à-dire le produit de ses dénégations, qui le hantent sans lui accorder aucune trêve logique. Dans ce « décor fantomatique ${ }^{29}$ », comme Philippe Jaworski l'a très justement remarqué, «Melville invite le lecteur à passer la limite qui sépare le sens littéral du sens métaphorique, opération qui suscite aussitôt l'attente et la recherche d'un sens ${ }^{30}$ ». Les objets vus «invraisemblables», tout comme les mots incompréhensibles ou obscurs recueillis par Delano, composent la dimension vertigineusement exotique - réaliste tout en étant inaccessible et trouble - animée par les figures d'Autrui, par la découverte d'un «nouveau monde» de signes. Nous retrouvons la trace d'une telle modélisation dans Taïpi, premier roman de Melville, véritable épreuve existentielle marquée par une «captive ethnograhy ${ }^{31}$ » et jalon fondateur de son inspiration où l'" "expérience du terrain" [...] nous est transmise à travers un double filtre : vécue, elle résulte d'un découpage effectué à partir d'un savoir préalable et constituant en quelque sorte une matrice de l'observation; écrite, elle est restituée par un discours qui la restructure $[\ldots]^{32} »$.

Observateur naïf et «de passage», le Delano de Melville entre impunément dans un espace où la rébellion est dissimulée par les esclavesacteurs dans les tréfonds de leur art de la mise en scène ${ }^{33}$. À ce propos, est à retenir l'image des

quatre nègres d'un certain âge, aux cheveux grisonnants, dont les têtes ressemblaient au noir sommet d'un saule écimé, offrant un vénérable

28. Cf. Sigmund Freud, «Le fétichisme», dans EEuvres complètes, t. XVIII, Paris, PUF, 1994 [1927], p. 125-131. Sur le thème de la dénégation en tant que notion pertinente dans une réflexion sur la fabrication d'objets ethno-muséographiques, je me permets de renvoyer à mon article : « Le goût de la croyance. Sur la dénégation nécessaire et son objet fétiche », L'Homme, 166 (2003), p. 171-184.

29. Herman Melville, Benito Cereno, op. cit., p. 67.

30. Philippe Jaworski, art. cit., p. 1090.

31. Jenny Franchot, «Melville's Traveling God », dans Robert S. Levine (dir.), The Cambridge Companion to Herman Melville, Cambridge, Cambridge University Press, 1998, p. 164.

32. Jean Laude, «Les Îles fortunées », L'Arc, 41 (1970), p. 4.

33. Cf. Sterling Stuckey, «The Tambourine in Glory: African Culture and Melville's Art », in Robert S. Levine (dir.), op. cit., p. 52. 
contraste avec le tumulte en contrebas, couchés qu'ils étaient, tels des sphinx, l'un sur le bossoir de tribord, un autre sur celui de bâbord, et le dernier couple face à face sur les pavois opposés, au-dessus des chaînes de hauban. Chacun tenait à la main des bouts de vieux cordages effrangés qu'il grattait, avec une sorte de satisfaction stoïque, pour en faire de l'étoupe, dont un petit amoncellement gisait à leur côté. Ils accompagnaient leur besogne d'une mélopée continue, produisant des bourdonnements et des vibrations analogues à ce que donne un groupe de cornemuseux grisonnants accompagnant un enterrement ${ }^{34}$.

Avec leur présence et leur mélopée, ces personnages, scandant un labeur anodin, sont censés calmer et tenir au respect de leurs rôles les plus jeunes parmi les révoltés. Une autre de ces activités de façade est jouée par les femmes esclaves participant avec leur bras et accompagnant avec leurs chants les manœuvres de mouillage dirigés par Delano qui est assisté par Babo dans son rôle officiel de «capitaine des esclave ${ }^{35}$ » dans le port naturel de l'île :

Les voiles déchiquetées et les vergues gauchies eurent tôt fait de reprendre leur assiette. Et point de bras ni de drisse qui ne fussent halés aux chants allègres des nègres pleins d'ardeur.

Les braves garçons, se dit le capitaine Delano, avec un peu d'entrâ̂nement ils feraient de bons marins. Voyez donc, même les femmes se mettent à tirer et à chanter elles aussi. Elles doivent faire partie de ces négresses Achantis qui font de si remarquables soldats, à ce qu'on dit ${ }^{36}$.

Ces scènes enrobées des allures de la normalité, masquent donc une subversion silencieuse ${ }^{37}$. Au sein de cette normalité théâtrale, tout en obligeant Benito Cereno à interpréter soi-même, sa condition «nécessaire » de maître à bord, les Noirs miment eux-mêmes, leur condition d'esclaves ainsi que leur situation objective de rescapés de la mer.

Les scènes données à voir à Delano nous parlent d'une stratégie esthétique et performative de la révolte que des conduites «habituelles»

34. Herman Melville, Benito Cereno, op. cit., p. 67.

35. Ibid., p. 131.

36. Ibid.

37. Cf. Benjamin D. Reiss, « Madness and Mastery in Melville's "Benito Cereno" », Criticism, XXXVIII/1 (1996), p. 119. 


\section{Fictions et visions littéraires d'un bateau négrier}

véhiculent et dissimulent en même temps. Au sujet des descriptions melvilliennes de cette construction artistique dissimulant et servant la conspiration, une notation de Stuckey apparaît significative : «In the novella, martial intent is hidden behind the rhythmic play of, a regular reminder to the Spaniards that the hatchets are in hand for more than musical purpose $e^{38}$ ». À travers la représentation d'une activité censée relever d'une ritualité ordinaire, ce rappel musical et secret est à la fois le langage de la menace adressée aux Espagnols et de la communication complice que les Africains entretiennent entre eux au cours de la durée suspendue inaugurée par la présence de Delano à bord du San Dominick :

Le gaillard d'arrière se terminait par une dunette ample et haute, à l'avant de laquelle, perchés comme les travailleurs d'étoupe à quelques pieds au-dessus de la populace, s'alignaient assis en tailleur à intervalles réguliers, six autres Noirs; chacun muni d'une hachette rouillée qu'il était occupé à fourbir, à l'aide d'un morceau de brique et d'un chiffon, à l'image d'un marmiton; tandis qu'entre chaque couple gisait une petite pile de hachette, dont les tranchants rouillés, tournés vers le dehors, attendaient la suite de l'opération. S'il arrivait aux quatre travailleurs d'étoupe de s'adresser brièvement à une ou des personnes de la foule en dessous, les six polisseurs de hachettes, eux, ne parlaient à personne ni n'échangeaient la moindre parole entre eux, mais restaient là, rivés à leur travail, sauf à certains moments où, avec cette prédilection caractéristique chez le nègre à unir industrie et passetemps, ils entrechoquaient deux à deux leurs hachettes telles des cymbales, en faisant un vacarme barbare ${ }^{39}$.

Tout comme le stéréotype ou le préjugé peuvent rarement faire l'objet d'un assaut frontal véritablement efficace, la mutinerie, tout en bouleversant l'ordre des choses, nécessite de lieux communs, de malentendus ou de feintes brouillant les apparences des rapports de force jusque-là établis. $\mathrm{Si}$, dès le début de la rencontre, Delano sert « d'interlocuteur privilégié à toutes ces langues empressées », alors que «son regard tout aussi empressé embrassait l'ensemble de ces visages et ces objets qui l'entouraient ${ }^{40}{ }$,

\footnotetext{
38. Sterling Stuckey, op. cit., p. 52.

39. Herman Melville, Benito Cereno, op. cit., p. 67-68.

40. Ibid., p. 66 .
} 
la «syllabe inconnue [qui] courait d'homme en homme, comme entre les poteaux d'un télégraphe qui crépite ${ }^{41} »$, qu'il tente en vain d'entendre, demeure jusqu'à la fin étanche à sa compréhension. Et pourtant, sur ce « volcan en sommeil ${ }^{42}$ », dans chaque séquence (avec son attirail d'objets) du théâtre dont il est l'observateur attentif, la vérité rampante de la situation pourrait être visible. À cet égard, Sterling Stuckey a analysé l'évolution dynamique de la mise en scène : on passe imperceptiblement du spectacle, où tous les acteurs utilisent les «éléments» symboliques et matériels de la fiction, au conflit. Cette situation est également significative du passage presque indécelable de la transmission d'attitudes prétendument « ancestrales » (dont les activités «quotidiennes » auxquelles les esclaves sont supposés vaquer à bord du San Dominick sont des échos) à la mise en situation de spectacle d'un passé africain perdu.

En même temps, le manque de neutralité de la scène du San Dominick, déjà définie par sa situation spécifique, amplifie la puissance évocatrice «pré-théâtrale ${ }^{43}$ » où l'action réelle croise l'action fictive. Pour les captifs révoltés, le fait de se masquer permettrait, en outre, d'incorporer la présence étrangère de Delano, à l'intérieur de leur univers culturel africain d'origine. Comme l'avait finement anticipé André Schaeffner, par rapport au dynamisme de la situation ethnologique dogon ${ }^{44}$, dans son texte «Rituel et pré-théâtre», le jeu de miroirs entre rite et changement participe de la mise en scène de la tradition qui est exposée par ses acteurs sociaux. Schaeffner, qui avait participé à la Mission ethnographique et linguistique Dakar-Djibouti (1931-1933), affirme de façon provocatrice : «À nombre de mythologues il a manqué de savoir danser, d'avoir ainsi entrevu en tout récit mythique un coin de spectacle, un théâtre embryonnaire ${ }^{45}$ ». À son avis les danseurs masqués dogon instituent une scène, tandis que les acteurs théâtraux entrent en scène. Les uns instaurent un théâtre, les autres en jouissent. La première forme révélerait un pré-théâtre c'est-à-dire une situation où la duplicité du théâtre relative à la présence d'êtres humains et de leurs simulacres est encore

\footnotetext{
41. Ibid., p. 112.

42. Ibid., p. 95.

43. André Schaeffner, «Rituel et pré-théâtre », dans Encyclopédie de la Pléiade, vol. 19 : Histoire des spectacles (G. Dumur, dir.), Paris, Gallimard, 1965, p. 21-54.

44. Cf. Gaetano Ciarcia, De la mémoire ethnographique. L'exotisme du pays dogon, Paris, Éditions de l'Ehess, 2003.

45. André Schaeffner, art. cit., p. 25.
} 


\section{Fictions et visions littéraires d'un bateau négrier}

plus évidente. Le manque de neutralité de la scène rituelle, déjà définie par sa situation spécifique, amplifierait la puissance évocatrice «préthéâtrale» où l'action réelle croise l'action fictive. Le fait de se masquer permettrait, en outre, d'incorporer les éléments du devenir, perçus comme des présences étrangères, à l'intérieur de l'univers «culturel» indigène. Ces notes semblent prolonger dans l'ethnologie ce que nous observons dans Benito Cereno : l'efficacité syncrétique de ceux qui manipulent le collage des signes du passé ne dérive d'un pur éclectisme mais bien du fait que leur action advient dans une arène locale où leur passé africain se déploie implicitement déjà comme une ressource politique «théâtrale ». L'action imaginée par Melville peut donc se traduire comme une affabulation dont les réalisateurs, suspendus entre des mondes «lointains », mais scandaleusement et férocement proches, interprètent l'actualité de leurs mémoires et de leurs aspirations conflictuelles. La spectacle des gestes qui deviennent performance (dans le sens que la langue anglaise attribue à ce mot) exprimerait le croisement des regards qui observent et s'observent, contribuant aux multiples possibilités de transfigurer la réalité, et donc finalement de la recréer.

Dans Benito Cereno, les observés jouent eux-mêmes, s'observent et se surveillent, dans une relation où les objets aux significations flottantes du récit deviennent des expédients pragmatiques. D'une manière inverse de l'idéal ethnographique classique de la participation au quotidien des autres prospectée par Bronislaw Malinowski, les actes et les objets inventés par Melville sont des faits apparemment «bruts» à partir desquels leur interprète, in situ et en papier, n'arrive pas à créer sa «mosaïque» de significations ${ }^{46}$. Tout en en amplifiant la portée historique, Benito Cereno finit par prédire la complexité dynamique et fuyante à l'œuvre sur les terrains où l'ethnographe est impliqué, souvent d'une manière dramatiquement circonstancielle, dans le travail scientifique de fabrication et d'inventaire des signes d'une situation.

46. Voir la présentation que Bronislaw Malinowski fait de sa «méthode » aux chapitres XVII et XVIII des Argonautes du Pacifique occidental, trad. de l'anglais par André et Simonne Devyer, Paris, Gallimard, 1963 [1922]. 